\title{
Apalutamide/Goserelin Regimen
}

National Cancer Institute

\section{Source}

National Cancer Institute. Apalutamide/Goserelin Regimen. NCI Thesaurus. Code

C160518.

A regimen consisting of apalutamide and goserelin that can be used for the treatment of prostate cancer. 